\title{
"Effect of Alkalinization on the Onset Action of Lignocaine in Epidural Anaesthesia"
}

\author{
Muhammad Mahmudul Haque $^{1^{*} \text {, Nitai Chandra Sarkar }}{ }^{2}$ \\ ${ }^{I}$ Assistant Professor, Department of Anaesthesiology and ICU, ShaheedZiaur Rahman Medical College \& \\ Hospital, Bogura, Bangladesh \\ ${ }^{2}$ Associate Prof. \& Head, Department of Anaesthesiology and ICU, Shaheedziaur Rahman Medical College \\ \&Hospital, Bogura, Bangladesh
}

*Corresponding Author: Muhammad Mahmudul Haque, 1Assistant Professor, Department of Anaesthesiology and ICU, ShaheedZiaur Rahman Medical College \& Hospital, Bogura, Bangladesh

\begin{abstract}
Lumbar epidural anaesthesia is one among the commonly performed regional anaesthesia especially for lower abdominal, lower limb surgeries and also for post-operative pain relief. Its ability to produce neuraxial blockade without dural puncture is an attractive option for spinal anaesthesia. To study the effect of alkalinization on the onset action of lignocaine in epidural anaesthesia. This study was done in ShaheedZiaur Rahman Medical College \& Hospital, Bogura, Bangladesh in the year of 2018-19. After the institutional ethical committee approval and informed consent from the patient, 100 patients posted for lower abdominal surgery were selected after inclusion and exclusion criteria. The inclusion criteria were ASA I\&II of both gender with an age group of 20-60 years and the exclusion criteria were age below 20years and above 60years, weight 45 to $85 \mathrm{kgs}$, spinal deformities, local sepsis, bleeding disorder and patient refusal. After the institutional ethical committee approval and informed consent from the patient, 100 patients posted for lower abdominal surgery were selected after inclusion and exclusion criteria and they were divided into two groups. Group $S$ received Lignocaine with adrenaline added with Saline and Group A received alkalized Lignocaine (with Sodium bicarbonate) with adrenaline. It was concluded that alkalinization shortens the onset of action of Lignocaine.
\end{abstract}

Keywords: Epidural anaesthesia, Alkalinization, Sodium bicarbonate, Saline, Lignocaine.

\section{INTRODUCTION}

Lumbar epidural anaesthesia is one among the commonly performed regional anaesthesia especially for lower abdominal, lower limb surgeries and also for post-operative pain relief. Its ability to produce neuraxial blockade without dural puncture is an attractive option for spinal anaesthesia [1-5]. Neural blockade follows when a local anesthetic is deposited in the vicinity of a nerve fiber. The onset of blockade occurs when the local anesthetic molecules reach the interior of the axoplasm in sufficient quantity. The drug molecule to reach the site of action, it has to travel many barriers especially in epidural anesthesia and so, the onset of action is delayed [6]. By modifying the physicochemical properties, we can quicken the onset of action of local anesthetic. Sodium bicarbonate is one such adjuvant that alters the same.

\subsection{Aim of the Study}

To compare the effect of alkalinization on the onset of action of alkalized and non-alkalized Lignocaine.

\section{MAterials ANd Methods}

This study was done in ShaheedZiaur Rahman Medical College \& Hospital, Bogura, Bangladesh in the year of 2018-19. After the institutional ethical committeeapproval and informed consent from the patient, 100 patients posted for lower abdominal surgery were selected after inclusion and exclusion criteria. The inclusion criteria were ASA I\&II of both gender with an age group of 20-60 years and the exclusion criteria were age below 20years and above 60years, weight 45 to $85 \mathrm{kgs}$, spinal deformities, local sepsis, bleeding disorder and patient refusal. The patients were divided into two groups. Group $(S)$ received Lignocaine with adrenaline added with Saline and Group (A) received alkalized Lignocaine (with Sodium bicarbonate) with adrenaline. Epidural block performed with 18-G Tuohy's epidural needle in L3-L4 interspace by loss of resistance technique. Test dose of $3 \mathrm{ml}$ of $2 \%$ lignocaine with adrenaline was injected followed by study drug 3 min later. Immediately after the injection, patients were placed in supine position. 
All patients were monitored for the onset of anesthesia, effects on cardiovascular and respiratory parameters, and intra- and postoperative complications. Pulse rate, mean blood pressure, and SPO2 recorded after injection of local anesthetic at interval of 5, 15, 30, 60, 90, 120 , and $150 \mathrm{~min}$ and more than $20 \%$ change as compared to preoperative values was considered significant.

\subsection{Onset of Sensory Analgesia}

Time from administration of drug to the time required for loss of touch sensation for an alcohol swab and loss of pinprick sensation at L2 level, which was judged at 1 min interval.

\subsection{Method of Alkalinization}

$0.8 \mathrm{ml}$ of $8.4 \%$ Sodium bicarbonate was added with each $10 \mathrm{ml}$ of $2 \%$ Lignocaine with the total of $20 \mathrm{ml}$. After the epidural space identification, the patients received the following drugs according to the group.All cases completed in Table1: Chart with Weight Distribution (Kgs) stipulated time. Data were collected, compiled, and tabulated. All results were expressed as mean \pm standard deviation. Two according to the drug received. The observations in the study are given as follows.

\subsection{Demographic Data}

There was not much significant variance in the each group.

\section{RESUlT}

This study was a randomized double blind study, conducted on a total of 100 patients grouped into two according to the drug received. The observations in the study are given as follows. In group N, the lowest onset time was 5 minutes and longest onset was 14 minutes with a mean of $7.87 \pm 1.834$ (standard deviation). In group $\mathrm{A}$, the, lowest onset of time was 1 minute and longest onset of time was 5 minutes with a mean of $2.665 \pm 0.994$ (standard deviation).

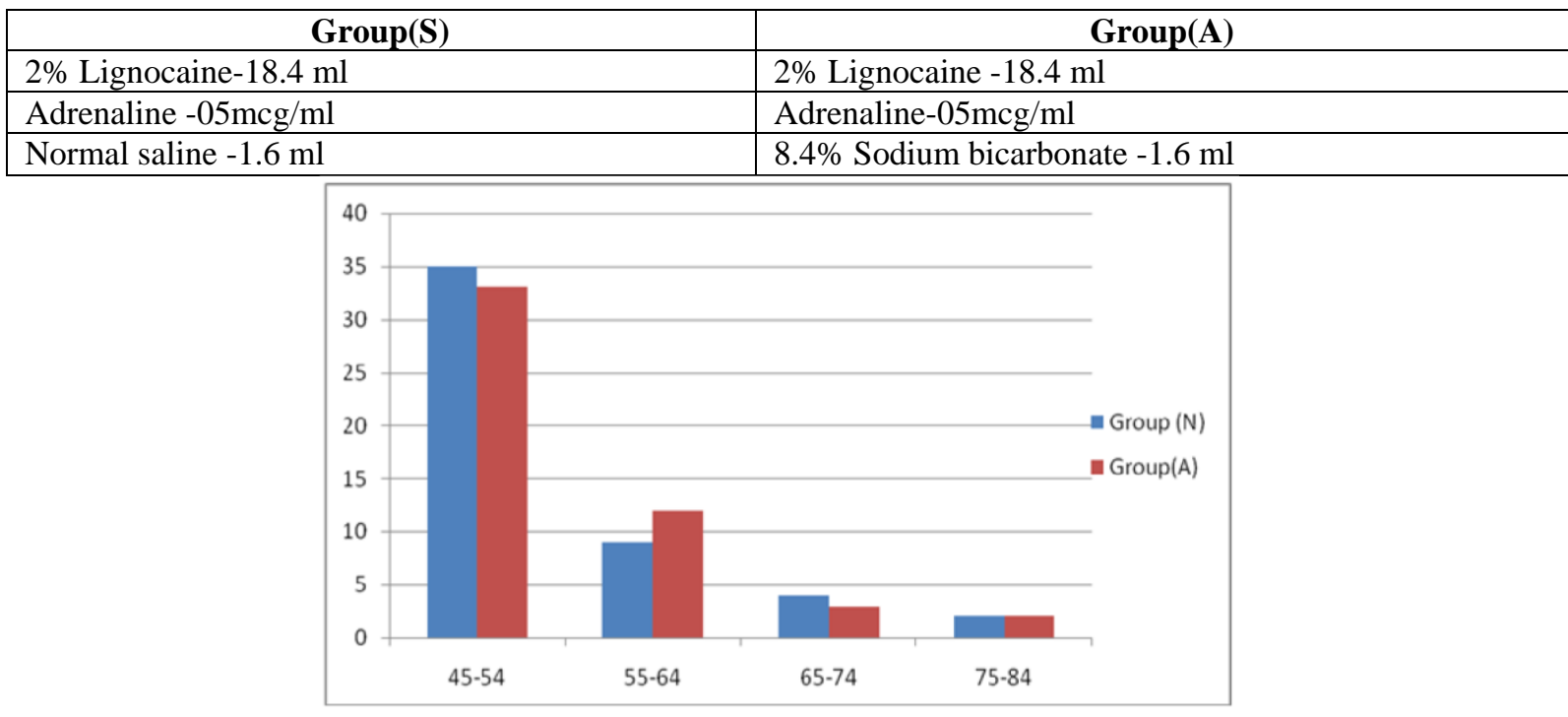

Fig1: Number of patients in left axis and weight in $\mathrm{kg}$ in right axis.

Table2: The Onset of Action in Minutes( $N=100)$

\begin{tabular}{|l|l|l|}
\hline \multicolumn{1}{|c|}{ Minutes } & Group(N) & \multicolumn{1}{c|}{$\operatorname{Group(A)}$} \\
\hline $0-2$ & - & 24 \\
\hline $2-4$ & - & 24 \\
\hline $4-6$ & 13 & 2 \\
\hline $6-8$ & 24 & - \\
\hline $8-10$ & 9 & - \\
\hline $10-12$ & 2 & - \\
\hline Total number & 50 & 50 \\
\hline
\end{tabular}

\section{DISCUSSION}

The $\mathrm{pH}$ of the local anesthetic solution immediately before injection to raise the $\mathrm{pH}$. This is called alkalinization of the solution. Anesthetic activity is dependent on having both the ionized and nonionized forms of the drug present after injection. Alkalinization increases the proportion of non-ionized drug and this could be advantageous. Care must be taken, because if too much alkali is added and mixing of any other drug, the local anesthetic will get precipitated. Local anesthetics are the basic 
drugs which have a pKa close to the normal extracellular $\mathrm{pH}$ of 7.4 , for example lignocaine has a $\mathrm{pKa}$ of 7.8. The drugs exist in two forms in the solution - the uncharged basic form (B) and the charged form $(\mathrm{BH}) . \mathrm{B}+\mathrm{H}+\leftrightarrow \mathrm{BH}$. The importance of the pKa-pH relationship is that this knowledge allows the calculation of the relative amounts of these two forms. When the $\mathrm{pH}$ is equal to the drug's pKa, $50 \%$ of the drug is in the uncharged form, and $50 \%$ is in the charged form. In acidic solutions, most of the drug will be in the charged form. When injected, the local anesthetic solution must be present in the tissues in both forms. The reason is that the drug has to diffuse to the site of action across several tissue barriers. The uncharged lipidsoluble form will diffuse across lipid barriers, for example, perineural sheath, or cell membrane. The charged water soluble form will diffuse across tissue fluid barriers, for example interstitial fluid. The site of action of the local anesthetic molecule is the inner (or cytoplasmic) end of the sodium channel in the cell membrane. The final pathway for all injected local anesthetics is to diffuse to the cell membrane (in the charged form) then reequilibrate to form both charged and uncharged forms adjacent to the outside of the nerve cell membrane. The molecules diffuse across the nerve cell membrane in the uncharged form then reequilibrate in the cytoplasm to have both forms present again. Next the charged form diffuses to and binds to its receptor on the inside of the transmembrane sodium channel. This binding results in a conformational change in the channel protein to block the passage of sodium ions into the cell in response to a subsequent action potential. Onset of sensory block was significantly quicker in alkalized group. Our observation was similar to observation by many authors [1-13]. Addition of bicarbonate for increasing $\mathrm{pH}$ of local anesthetic in epidural blockade hastens the onset of action by increasing the $\mathrm{pH}$ of the solution and thereby it increases the uncharged base form available for penetration. Our result is similar to Aparna AbhijitBagle et al. [11]. For epidural anaesthesia, time to achieve surgical anaesthesia is important. Onset of epidural anaesthesia was quicker with alkalized local anaesthetic solutions, but only by a few minutes. Dr.P.Chalapathy et al. concluded alkalinized lignocaine hydrochloride solution provides a significant advantage over non alkalinized lignocaine hydrochloride in terms of quicker onset, duration and quality of block [13]. Cardiovascular stability was found in all groups which is the feature of epidural analgesia and there was no incidence of postoperative complications in any of the group in our study.

\section{CONCLuSiON}

It was concluded that alkalinization shortens the onset of action of Lignocaine. We conclude that $\mathrm{pH}$-adjusted $1 \%$ lidocaine offers the advantage of a more rapid onset of sensory blockade, while motor blockade and the distribution of anesthesia are unaffected by $\mathrm{pH}$ change in epidural anesthesia.

\section{REFERENCES}

[1] AparnaAbhijitBagle, Satish Deshpande, Nagnath L Garthe. Effect of increase in pH of local anaesthetics on quality of epidural anesthesia. 2016; 2(2).

[2] Capogna G. Celleno D. Costantino P. Muratori F. Sebastiani M. Baldassini M. Canadian Journal of Anaesthesia Alkalinization improves the quality of lidocaine-fentanyl epidural anaesthesia for Caesarean section. 1993; 40(5):425-430.

[3] Gosteli P, Van Gessel E, Gamulin Z. Effects of $\mathrm{pH}$ adjustment and carbonation of lidocaine during epidural anesthesia for foot or ankle surgery. AnesthAnalg. 1995; 81:104-9.

[4] Milner QJ, Guard BC, Allen JG. Alkalinization of amide local anaesthetics by addition of $1 \%$ sodium bicarbonate solution. European journal of anaesthesiology. $2000 \mathrm{Jan} ; 17(1): 38-42$.

[5] Fulling PD, Peterfreund RA. Alkalinization and precipitation characteristics of $0.2 \%$ ropivacaine. RegAnesth Pain Med. 2000; 25: 518-21.

[6] Robinson J, Fernando R, Sun Wai WY, Reynolds F. Chemical stability of bupivacaine, lidocaine and epinephrine in $\mathrm{pH}$-adjusted solutions. Anaesthesia. 2000;55:853-8

[7] Milner QJ, Guard BC, Allen JG. Alkalinization of amide local anaesthetics by addition of $1 \%$ sodium bicarbonate solution. Eur J Anaesthesiol. 2000;17:38-42

[8] Tackley RM, Coe AJ. Alkalinised bupivacaine and adrenaline for epidural Caesarean section: A comparison with $0.5 \%$ bupivacaine. Anaesthesia. 1988 Dec; 43(12):1019-21.

[9] Kaufman JJ, Semo NM, Koski WS. Microelectrometric titration measurement of the pKa's and partition and drug distribution coefficients of narcotics and narcotic antagonists and their $\mathrm{pH}$ and temperature dependence. Journal of Medicinal Chemistry. 1975 Jul; 18(7):647-55.

[10] Benhamou D, Perrachon N, Labaille T, Bonhomme L. Alkalinization of epidural $0.5 \%$ 
bupivacaine for cesarean section. RegAnesth Pain Med. 1989 Sep 1; 14(5):240-3.

[11] Parnass SM, Curran MJ, Becker GL. Incidence of hypotension associated with epidural anesthesia using alkalinized and nonalkalinizedlidocaine for cesarean section. Anesthesia and analgesia. 1987 Nov; 66(11): 11 48-50.
[12] Dr. Chalapathy P, Dr. Sangeetha Rao D.Comparitive Study of The Effect of Lignocaine Hydrochloride With Adrenaline And It's Combination With Alkali Solution on Supraclavicular Brachial Plexus Block.

[13] Mehta R. Effect of alkalinization of lignocaine hydrochloride on brachial plexus block.2013.

Citation: Muhammad Mahmudul Haque, Nitai Chandra Sarkar, "Effect of Alkalinization on the Onset Action of Lignocaine in Epidural Anaesthesia”. ARC Journal of Anesthesiology. 2020; 5(2):22-25. DOI: doi.org/ 10.20431/2455-9792.0502005.

Copyright: (C) 2020 Authors. This is an open-access article distributed under the terms of the Creative Commons Attribution License, which permits unrestricted use, distribution, and reproduction in any medium, provided the original author and source are credited. 